\title{
Photon acceleration as a scattering process
}

\author{
JT Mendonça and R Bingham
}

\section{Published version information}

Citation: Mendonça JT and Bingham R. "Photon Acceleration as a Scattering Process." Plasma Physics and Controlled Fusion, vol.57 no.4 (2015): 044011 doi.org/10.1088/0741-3335/57/4/044011

This version is made available in accordance with publisher policies. Please cite only the published version using the reference above. 


\title{
Photon Acceleration as a Scattering Process
}

\author{
J.T. Mendonça* \\ IPFN, Instituto Superior Técnico,1049-001 Lisboa, Portugal and \\ Instituto de Física, Universidade de São Paulo, São Paulo SP, 05508-090 Brasil \\ R. Bingham \\ STFC Rutherford Appleton Laboratory, \\ Didcot, Oxfordshire OX11 OQX, UK
}

\begin{abstract}
The concept of photon acceleration is revisited. It is shown here than it can described as an inelastic scattering process. This new approach is applied to the study of relativistic plasma bubbles or wakefields, as those created by an intense laser pulse or by a particle beam moving in a background plasma. Scattering of an incident probe photon beam by a relativistic plasma density perturbation is considered. The angular dependence of frequency and intensity of the scattered signal are discussed in detail. The case of relativistic electron beams propagating in vacuum is also considered for comparison. Such a scattering process could be used as a diagnostic of the space-time structures created by laser driven bubbles and wakefields, for particle acceleration studies.
\end{abstract}

PACS numbers:

*Electronic address: titomend@ist.utl.pt

${ }^{\dagger}$ Electronic address: bob.bingham@stfc.ac.uk 


\section{INTRODUCTION}

Photon acceleration is a generic optical process which takes place in plasmas and in other optical media, and includes a variety of phenomena such as photon trapping by electron plasma waves, and self-phase and cross-phase modulation in optical fibers [1]. It is well known that it can lead to significant frequency upshifts. This occurs when a probe photon beam interacts with relativistic perturbations, created by moving ionization fronts $[2,3]$ or by moving nonlinear perturbations, as those created by intense laser pulses in the medium $[4,5]$. These two cases are similar but physically distinct, and they have both been confirmed experimentally. Photon acceleration by relativistic ionization fronts has be observed in microwave experiments [6], as well as in optical experiments where a proof of principle was established [7]. More recently, photon trapping effects were identified in laser wakefield experiments [8], similar to those used for laser acceleration [9].

Photon acceleration is also related to sophisticated kinetic effects, most particularly, to photon Landau damping of electron plasma waves. For plasma waves with relativistic phase velocities, as those created by a laser pulse, electron Landau damping is negligible, but photon Landau damping can be significant. This kinetic process was first proposed using geometric optics and a phenomenological photon kinetic equation [10], and then confirmed by a full wave description of electromagnetic radiation [11]. It is also interesting to notice that photon Landau damping shows striking similarities with electron Landau damping in the quantum plasma regime [12]. Such kinetic processes, although conceptually relevant and innovative, have not yet been experimentally addressed.

A variety of theoretical descriptions can be used to describe photon acceleration, from geometric ray tracing to full wave calculations, using both classical and quantum methods [1]. An additional and different view of this process is proposed here. We show that, in certain configurations, photon acceleration can also be seen as an inelastic photon scattering. This could be particularly relevant to diagnose the velocity and shape of relativistic bubbles and wakefield structures, excited by intense laser pulses or by short particle beam moving across the plasma.

We first consider photon scattering by a relativistic bubble with arbitrary shape, moving in a background isotropic plasma and driven by an intense laser pulse, or by a particle beam. Our scattering model, the resulting angular dependence of the scattered frequency shifts and 
scattered efficiencies will be discussed. We then compare the laser driven bubble with the case of a bunch of charged particles moving in vacuum.

\section{WAVE SCATTERING MODEL}

Let us assume a relativistic bubble, with arbitrary shape, moving in a plasma with velocity u. The electron plasma density can be described by

$$
n=n_{0}[1-\epsilon F(\mathbf{r}-\mathbf{u} t)]
$$

where $n_{0}$ is the equilibrium plasma density, $\epsilon=\delta n / n_{0}$ is the perturbation amplitude associated with the bubble, and $F(\mathbf{r}-\mathbf{u} t)$ is a generic shape function of the moving perturbation, to be specified later.

The incident wave, associated with a probe photon beam, can be described by its vector potential

$$
\mathbf{A}_{i}(\mathbf{r}, t)=\mathbf{A}_{0} \exp \left(i \mathbf{k}_{0} \cdot \mathbf{r}-i \omega_{0} t\right)+\text { c.c. }
$$

The interaction of this incident wave with the moving bubble creates an electron current, given by

$$
\mathbf{J}=-e n \mathbf{v}, \quad \mathbf{v}=\frac{e}{m}\left(\mathbf{A}_{i}+\mathbf{A}_{s}\right)
$$

where $-e$ and $m$ are the electron charge and mass, and the vector potential $\mathbf{A}_{s}$ describes the scattered wave. This current can then be written as

$$
\mathbf{J}=-\frac{e^{2} n_{0}}{m}\left[\mathbf{A}_{s}-\epsilon F(\mathbf{r}-\mathbf{u} t) \mathbf{A}_{i}\right]
$$

The scattered wave will then be determined by the wave equation

$$
\left(\nabla^{2}-\frac{1}{c^{2}} \frac{\partial^{2}}{\partial t^{2}}\right) \mathbf{A}_{s}=-\mu_{0} \mathbf{J}
$$

where $\mu_{0}$ the magnetic permeability of vacuum. At this point, it is useful to introduce a time Fourier transformation of the scattered vector potential, such that

$$
\mathbf{A}_{s}=\int \mathbf{A}_{\omega}(\mathbf{r}) \exp (-i \omega t) \frac{d \omega}{2 \pi}
$$

The wave equation (5) then becomes $\left(\nabla^{2}+k^{2}\right) \mathbf{A}_{\omega}=-\mu_{0} \mathbf{J}_{\omega}$, with the current spectral component given by

$$
\mathbf{J}_{\omega}=\epsilon \frac{\omega_{p}^{2}}{\mu_{0} c^{2}} \mathbf{A}_{0} \int F(\mathbf{r}-\mathbf{u} t) e^{i \omega t}\left\{e^{i \mathbf{k}_{0} \cdot \mathbf{r}-i \omega_{0} t}+\text { c.c. }\right\} d t
$$


where $\omega_{p}$ is the equilibrium plasma frequency, and the wave number of the scattered wave $k$ is determined by the linear dispersion relation $k^{2} c^{2}=\omega^{2}-\omega_{p}^{2}$. Let us now consider the following decomposition of the bubble profile $F(\mathbf{r}-\mathbf{u} t)=f\left(\mathbf{r}_{\perp}\right) g(\eta)$, with $\eta=x-u t$. where $f\left(\mathbf{r}_{\perp}\right)$ and $g(\eta)$ pertain for the perpendicular and parallel (with respect to the bubble motion) bubble profiles. Without loss of generality we have assumed here that the bubble moves along the x-coordinate, $\mathbf{u}=u \mathbf{e}_{x}$. We now introduce a Fourier transformation of the parallel shape function, as

$$
g(\eta)=\int_{-\infty}^{\infty} g(q) \exp (i q \eta) \frac{d q}{2 \pi}
$$

Two simple but useful models can be used for $g(\eta)$. The first one is a Gaussian shape of width $\sigma$, with the corresponding Fourier transformation

$$
g(\eta)=\exp \left(-\frac{\eta^{2}}{2 \sigma^{2}}\right), \quad g(q)=2 \pi \sigma \exp \left(-\frac{1}{2} q^{2} \sigma^{2}\right)
$$

It is well known that wakefields produced by an intense laser pulse, in the so-called bubble regime [17], have a nearly spherical shape [18, 19], with a strong electron depletion. A super-Gaussian shape would probably be more adequate to describe the bubble, but the final frequency shift of photons interacting with such moving structures is not very much sensitive to the actual shape of the electron density depletion, and is mainly determined by the electron density minimum and by the bubble velocity [1]. The use of this simple Gaussian profile is therefore justified. Alternatively, we can use a cosine function

$$
g(\eta)=[1-H(|\eta|-\pi \sigma / 2)] \cos (\eta / \sigma)
$$

where $H(x)$ is the Heaviside function, and the Fourier components are determined by the simple integral

$$
g(q)=2 \int_{0}^{\pi / 2} \cos x \exp (i q \sigma x) d x
$$

This simple cosine model can accurately describe a laser wakefield for moderate laser intensities, below the bubble regime. Instead of an isolated bubble, or a simple cosine wake, we could equally consider a sequence of bubbles associated with a more complex wakefield structure. It was shown recently that donut wakefields could be excited in a plasma, using a laser with orbital angular momentum [20]. In the high intensity laser regime, the corresponding toroidal bubble is able to accelerate positron beams [21]. Replacing the Fourier development (8) in the wave equation $(7)$, we can then derive

$$
J_{\omega}=\epsilon \frac{\omega_{p}^{2}}{\mu_{0} c^{2}}\left[S_{+}\left(\mathbf{r}_{\perp}\right) e^{i\left(q_{+} \mathbf{e}_{x}+\mathbf{k}_{0}\right) \cdot \mathbf{r}}+S_{-}\left(\mathbf{r}_{\perp}\right) e^{i\left(q_{-} \mathbf{e}_{x}-\mathbf{k}_{0}\right) \cdot \mathbf{r}}\right]
$$


where we have defined the following auxiliary quantities

$$
S_{ \pm}\left(\mathbf{r}_{\perp}\right)=\left(\mathbf{A}_{0} \cdot \mathbf{e}_{\omega}\right) \frac{f\left(\mathbf{r}_{\perp}\right)}{u} g\left(q_{ \pm}\right), \quad q_{ \pm}=\frac{1}{u}\left(\omega \mp \omega_{0}\right)
$$

with $\mathbf{e}_{\omega}=\mathbf{A}_{\omega} / A_{\omega}$. This will allow us to determine the intensity and spectrum of the scattered radiation, as discussed next.

\section{SCATTERED RADIATION}

Let us now consider a given direction of propagation for the scattered wave signal, as determined by the wavevector $\mathbf{k}$, such that $\mathbf{e}_{\omega} \cdot \mathbf{k}=0$. The solution of equation (12) gives the scattered field observed at a generic position $\mathbf{r}$, as

$$
\begin{array}{r}
A_{\omega}(\mathbf{r})=-\frac{\epsilon \omega_{p}^{2}}{2 k c^{2}} e^{i \mathbf{k} \cdot \mathbf{r}} \int^{\mathbf{r}} d \mathbf{r}^{\prime} \int \frac{d \mathbf{q}_{\perp}}{(2 \pi)^{2}} \times \\
\times e^{-i \mathbf{k} \cdot \mathbf{r}^{\prime}} \sum_{ \pm}\left\{S_{ \pm}\left(\mathbf{q}_{\perp}\right) e^{i\left(\mathbf{q}_{p} m \pm \mathbf{k}_{0}\right) \cdot b f r^{\prime}}\right\}
\end{array}
$$

where $S_{ \pm}\left(\mathbf{q}_{\perp}\right)$ results from an additional Fourier transformation in the trasnverse coordinate direction, as defined by

$$
f\left(\mathbf{r}_{\perp}\right)=\int f\left(\mathbf{q}_{\perp}\right) \exp \left(i \mathbf{q}_{\perp} \cdot \mathbf{r}_{\perp}\right) \frac{d \mathbf{q}_{\perp}}{(2 \pi)^{2}}
$$

The asymptotic value of the radiation field, which can be measured far away from the interaction zone, can be obtained by assuming $|\mathbf{r}| \rightarrow \infty$ in the above integration. We therefore get, for the dominant spectral components

$$
A_{\omega}(\mathbf{r}) \simeq-\frac{\pi \epsilon \omega_{p}^{2}}{k c^{2}} e^{i \mathbf{k} \cdot \mathbf{r}} \sum_{ \pm} S_{ \pm}\left(\mathbf{q}_{\perp}\right) \delta\left(\mathbf{q}_{ \pm}-\left(\mathbf{k}-\mathbf{k}_{0}\right)\right)
$$

where the relevant wavevectors of the bubble structure components are $\mathbf{q}_{ \pm}=q_{ \pm} \mathbf{e}_{x}+\mathbf{q}_{\perp}$. This defines the wavevectors of the allowed scattered radiation as

$$
\mathbf{k}=\mathbf{q}_{ \pm} \pm \mathbf{k}_{0} \equiv \frac{1}{u}\left(\omega \mp \omega_{0}\right) \mathbf{e}_{x}+\mathbf{q}_{\perp} \pm \mathbf{k}_{0}
$$

Using the dispersion relation for the scattered waves, we can write this expression in terms of the radiated frequency $\omega$, as

$$
\frac{\omega^{2}}{\gamma_{b}^{2}} \mp 2 \omega \omega_{0}\left(1-\frac{\mathbf{k}_{0} \cdot \mathbf{u}}{\omega_{0}}\right)+\omega_{0}^{2}\left(\frac{u^{2}}{c^{2}}-2 \frac{\mathbf{k}_{0} \cdot \mathbf{u}}{\omega_{0}}\right)+Q_{\perp}^{2} u^{2}=0
$$


where we have used $Q_{\perp}^{2}=q_{\perp}^{2} \pm 2\left(\mathbf{q}_{\perp} \cdot \mathbf{k}_{0 \perp}^{2}\right)$. We have also introduced the relativistic gamma factor of the moving bubble, as $\gamma_{b}=\left(1-u^{2} / c^{2}\right)^{-1 / 2}$. The solution is

$$
\omega= \pm \omega_{0} \gamma_{b}^{2}\left(1-\frac{\mathbf{k}_{0} \cdot \mathbf{u}}{\omega_{0}}\right)\{1 \pm \xi\}
$$

where the two plus and minus signs have to be taken independently, and the quantity $\xi$ is determined by

$$
\xi^{2}=1-\frac{\left[\left(1+Q_{\perp}^{2} c^{2} / \omega_{0}^{2}\right) u^{2} / c^{2}-2 \mathbf{k}_{0} \cdot \mathbf{u} / \omega_{0}\right]}{\left(1-\mathbf{k}_{0} \cdot \mathbf{u} / \omega_{0}\right)^{2} \gamma_{b}^{2}}
$$

It is clear from this result that the scattered frequency $\omega$ will depend on the direction and magnitude of the bubble velocity, as well as on of the direction of propagation of the incident and scattered radiation, as determined by $\mathbf{k}_{0}$ and $\mathbf{k}$. Very large frequency shifts $\Delta \omega \equiv\left(\omega-\omega_{0}\right) \gg \omega_{0}$ can eventually occur. It is interesting to note that this expression for the scattered frequency strongly resembles the result obtained independently by Mori [13] and by one of us [1] for the "dark source", where radiation is generated by a plasma perturbation moving across a static electric field. Here the static field is replaced by the incident wave. Before discussing the frequency shifts in detail, it is useful to consider the amplitude of the scattered radiation, as determined by equation (16). This leads to the following form factor, characterizing the intensity of the scattering radiation.

$$
S\left(\omega, \omega_{0}\right) \equiv \frac{\left|A_{\omega}\right|^{2}}{\left|A_{0}\right|^{2}}=\left(\frac{\pi \epsilon \omega_{p}^{2}}{k u c^{2}}\right)^{2}\left|\left(\mathbf{e}_{0} \cdot \mathbf{e}_{\omega}\right)\right|^{2}\left|F\left(\mathbf{q}_{\perp}, q_{ \pm}\right)\right|^{2}
$$

where $F\left(\mathbf{q}_{\perp}, q_{ \pm}\right) \equiv f\left(\mathbf{q}_{\perp}\right) g\left(q_{ \pm}\right)$. In order to be more specific, it is useful to consider the case of a Gaussian bubble, defined by

$$
F\left(\mathbf{q}_{\perp}, q_{ \pm}\right)=\exp \left(-\frac{\eta^{2}}{2 \sigma^{2}}-\frac{r_{\perp}^{2}}{2 \sigma_{\perp}^{2}}\right)
$$

The intensity form factor becomes

$$
\begin{aligned}
S\left(\omega, \omega_{0}\right) & \equiv \frac{\left|A_{\omega}\right|^{2}}{\left|A_{0}\right|^{2}}=\frac{2^{3} \pi^{5} \epsilon^{2} \omega_{p}^{4}}{k^{2} u^{2} c^{4}}\left|\left(\mathbf{e}_{0} \cdot \mathbf{e}_{\omega}\right)\right|^{2} \times \\
& \times \sigma^{2} \sigma_{\perp}^{2} \exp \left[-q_{ \pm}^{2} \sigma^{2}-\left(\mathbf{k} \mp \mathbf{k}_{0}\right)_{\perp}^{2} \sigma_{\perp}^{2}\right]
\end{aligned}
$$

We notice here that the scattered amplitudes are only significant for $\left|q_{ \pm}\right| \leq 1 / \sigma$, and $\left|q_{\perp}\right| \leq 1 / \sigma_{\perp}$. We will come back to this later. It is now useful to discuss equation (20) for some specific condition. First, we consider the explicit angular dependence of the scattered frequencies, by defining a scattering angle $\theta$ such that

$$
\mathbf{k}_{0} \cdot \mathbf{u}=-k_{0} u \cos \theta=-\beta \cos \theta \sqrt{\omega_{0}^{2}-\omega_{p}^{2}}
$$


with $\beta=u / c$. Equation (20) becomes

$$
\omega=\omega_{0} \gamma_{b}^{2}\left(1+\beta \cos \theta \sqrt{1-\frac{\omega_{p}^{2}}{\omega_{0}^{2}}}\right)\{1 \pm \xi\}
$$

For high frequency incident waves, such that $\omega_{0}^{2} \gg Q_{\perp}^{2} c^{2}, \omega_{p}^{2}$, the quantity $\xi$ can be given by the simple expression

$$
\xi^{2}=1-\frac{\beta^{2}+2 \beta \cos \theta}{\gamma_{b}^{2}(1+\beta \cos \theta)^{2}}
$$

This shows that, for large relativistic factors $\gamma_{b} \gg 1$ and for counter-propagation, such that $\theta \sim 0$, we have very high frequencies for the scattered radiation. In contrast, the scattered frequencies are lower for forward propagation of the incident wave, such that $\theta \sim \pi$. This is illustrated in figure 1, where the upper frequency branch of the scattered photons is represented. The lower branch is less sensitive to the change in the bubble gamma factor, and background plasma frequency, and is not relevant to diagnostic purposes.

An interesting and somewhat surprising situation occurs when the incident wave propagates at right angles with respect to the bubble velocity, $\theta \sim \pm \pi / 2$. In this case the scattered frequency is approximately given by

$$
\omega \simeq \omega_{0} \gamma_{b}^{2}\left[1 \pm \sqrt{1-\frac{\beta^{2}}{\gamma_{b}^{2}}}\right]
$$

For $\gamma_{b} \gg 1$ this leads to high and low frequency branches, or the order of $\omega_{\text {high }} \simeq 2 \omega_{0} \gamma_{b}^{2}$, and $\omega_{\text {low }} \simeq \omega_{0} / 2$, a sub-harmonic of the incident wave. It should however be noticed that high frequency shifts, such that $\omega \gg \omega_{0}$ imply that $q_{ \pm} \sim \omega / u$, and are usually associated with low scattered intensities, because the efficiency decays exponentially for high values of $q_{ \pm}$, as shown by equation (23). We can see that scattering is stronger for low frequencies, and decays for $\omega \gg u / \sigma$. However the situation changes if we have electron structures inside the bubble, which we have neglected in our simple analysis but are known to occur in the bubble regime of laser acceleration. Furthermore, the present model is based on the first Born approximation, which implies that the incident wave is not strongly modified by the existence of the bubble. A nonlinear scattering model will eventually lead to an increase of efficiency for the high frequency scattered radiation. 


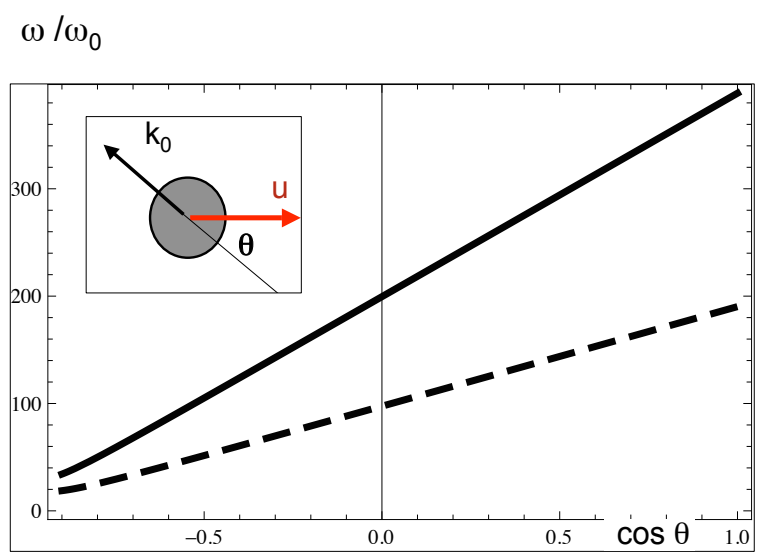

FIG. 1: Higher frequency part of the scattered spectrum, as a function of the angle between the bubble velocity $\mathbf{u}$ and the incident wavevector $\mathbf{k}_{0}$, for $\gamma_{b}=10$, and $\gamma_{b}=7$ (dashed). We have used $\omega_{p}^{2} / \omega_{0}^{2}=0.1$

\section{RELATIVISTIC PARTICLE BUNCH}

It is useful to compare the previous case of a plasma density perturbation of the bubble type, which can move at relativistic speed through a plasma without implying the relativistic motion of the individual plasma particles, with that of a beam of charged particles which are actually moving at relativistic speed in vacuum. It is known that the electrons inside a wakefield are nearly at rest with respect to the laboratory frame, because they are mainly pushed by the driving laser pulse towards to boundaries of the plasma density perturbation. In a typical laser wakefield experiment, only a small fraction of the electron density population is actually self-injected and accelerated $[9,17]$. This means that, although the bubble moves with relativistic velocity, the dominant part of the electron population stays at rest, on the average. In contrast, if the perturbation is due to an electron beam, the entire bunch of electrons moves with relativistic speed.

For a bunch of $N$ electrons with velocity $\mathbf{u}$ and density profile $F(\mathbf{r}-\mathbf{u} t)$, equation (1) is replaced by

$$
n=n_{b} F(\mathbf{r}-\mathbf{u} t), \quad N=\int n_{b} d \mathbf{r}
$$

where $n_{b}$ is the beam density, and a background vacuum is assumed, $n_{0}=0$. For a low intensity incident wave, the electron energy can be approximately assumed as unperturbed 
and equal to $m \gamma_{b} c^{2}$, and the scattering current is determined by

$$
\mathbf{J}=-\frac{e^{2} n_{b}}{m \gamma_{b}} \mathbf{A}_{i} F(\mathbf{r}-\mathbf{u} t)
$$

Repeating the above calculations to the present situation, we arrive at an expression for the scattered frequencies, which takes the form

$$
\left[\frac{1}{u}\left(\omega \mp \omega_{0}\right) \pm k_{0 x}\right]^{2}+\left(\mathbf{q}_{\perp} \pm \mathbf{k}_{0}\right)^{2}=\frac{\omega^{2}}{c^{2}}
$$

Solving for the frequency, and using (24), we get a result which only differs from (25) because the plasma frequency is zero, $\omega_{p}^{2}=0$. On the other hand, the form factor for the scattered energy becomes

$$
S\left(\omega, \omega_{0}\right)=\frac{\pi^{2} \omega_{b}^{4}}{k^{2} u^{2} c^{4}} \frac{\left|\left(\mathbf{e}_{0} \cdot \mathbf{e}_{\omega}\right)\right|^{2}}{\gamma_{b}^{2}}\left|F\left(\mathbf{q}_{\perp}, q_{ \pm}\right)\right|^{2}
$$

where $\omega_{b}$ is the beam plasma frequency. The main difference with respect to equation (23) is the appearance of a factor $\gamma_{b}^{2}$ in the denominator. This is due to an increase of the effective mass for the electrons in the beam, which strongly reduces the scattering current and consequently the scattered energy, with respect to the case of a plasma bubble where the electrons are at rest. It should be noticed that the two kinds of photon scattering processes considered here, from a bubble or from an electron beam, can occur simultaneously in the laser acceleration in the bubble regime, where a short electron pulse, with an internal width much smaller than the laser waist and bubble width, is excited inside the plasma bubble. In this case, the scattered fields and spectra associated with these two moving perturbations will superpose.

\section{CONCLUSIONS}

We have shown here that photon acceleration can be seen as a particular case of inelastic scattering process, where probe photons bounce back with increased energy from a moving density perturbation. We have illustrated this idea by studying the interaction of a probe photon beam with a relativistic bubble moving in a plasma, produced for instance by an intense laser laser pulse used for laser acceleration studies [9, 14]. We have derived expressions for the angular dependence of the scattered frequency, and for the corresponding scattered intensities. We have also compared the relativistic bubble case with that of a bunch of relativistic electrons moving in vacuum. 
It should be noticed that, in our model, inelastic scattering was only seen from the point of view of the probe photons, and momentum conservation of the moving perturbation was considered. This is valid when the total momentum carried by the relativistic bubble or electron bunch is much larger than that of the probe photons. In plausible experimental configurations, the relativistic bubble is excited by ultra-intense laser beams (in the range of tens of Tera-Watt), whereas the probe beam intensity is a few orders of magnitude smaller thus justifying the neglect of recoil effects on the relativistic bubble. The same is true for the relativistic electron bunch, where the average electron momentum is much higher than the probe photon momentum, because the inequality $\gamma_{b} \gg \lambda_{C} k$, where $\lambda_{C}$ is the Compton wavelength and $k$ the incident photon wavenumber, is always satisfied.

We suggest that such an upshifting frequency scheme could be used to diagnose the space-time structure of nonlinear perturbations moving in a plasma, in particular their mean velocity and shape. This is of particular relevance to the project AWAKE, now being implemented at CERN, which proposes to create plasma wakefields using high energy proton beams [22].

Although with a lower efficiency, the same diagnostic technique could also be applied to measure similar properties of particle bunches moving in vacuum. A similar approach could be used to study photon scattering by nonlinear perturbations in a generic optical medium. In particular, the photon acceleration process described here could eventually explain the recent observations of a presumed classical analogue to Hawking radiation [23, 24]. In our view, the observed radiation spectrum is simply due to side scattering of the incident laser pulse by its own relativistic nonlinear perturbation.

In this work we have use a simple theoretical approach, based on the first Born approximation. We expect that a more detailed nonlinear study will confirm the frequency shifts of the scattered radiation derived here, and reveal increased scattering efficiencies in the nonlinear regime. We have also neglected other photon acceleration processes which can be associated with radiation trapped inside the bubble [15], or transmitted across it [16] . The integrated influence of all these aspects will be studied with the help of pic and photon kinetic code simulations in a future publication.

To our knowledge, photon scattering diagnostics of relativistic laser wakefield or electron beams have not yet been developed. Our results, and in particular the two branches of frequency upshifts given by eqs. (25) or (27), could be used to inspire new and innovative 
experiments in the field of laser-plasma interactions.

[1] J.T. Mendonça, Theory of Photon Acceleration, Institute of Physics Publishing, Bristol (2001).

[2] V.I. Semenova, Sov. J. Radiophys. Quantum Electron., 10, 599 (1967).

[3] M. Lampe, E. Ott and J.H. Walker, Phys Fluids, 10, 42 (1978).

[4] J.T. Mendonça, J. Plasma Phys., 22, 15 (1979).

[5] S.C. Wilks et al, Phys. Rev. Lett., 62, 2600 (1989).

[6] R.L. Savage Jr., C. Jishi and W.B. Mori, Phys. Rev. Lett., 68, 946 (1992).

[7] J.M. Dias et al, Phys. Rev. Lett., 78, 4773 (1997).

[8] C.D. Murphy et al. Phys. Plasmas, 13, 033108 (2006).

[9] S.P.D. Mangles, C.D. Murphy, Z. Najmudin et al., Nature, 431, 535 (2004).

[10] R. Bingham, J.T. Mendonça and J.M. Dawson, Phys. Rev. Lett., 78, 247 (1997).

[11] J.T. Mendonça and A. Serbeto, Phys. Plasmas, 13, 102109 (2006).

[12] G. Brodin, J. Zamanian and J.T. Mendonça, submitted to Phys. Rev. E, (2014).

[13] W.B. Mori, T. Katsouleas, J.M. Dawson and C.H. Lai, Phys. Rev. Lett., 74, 542 (1995).

[14] R. Bingham, J.T. Mendonça and P.K. Shukla, Plasma Phys. Control. Fusion, 46, R-1 (2004).

[15] J.T. Mendonça, New J. Phys., 11, 013029 (2009).

[16] J.T. Mendonça and K. Hizanidis, Plasma Phys. Contr. Fusion, 54, 035006 (2012).

[17] A. Pukhov and J. Meyer ter Vehn, Appl. Phys. B, 74, 355 (2002).

[18] I. Kostyukov, A. Pukhov and S. Kiselev, Phys. Plasmas, 11, 5256 (2004).

[19] W. Lu, C. Huang, M. Zhou, W.B. Mori and T. Katsouleas, Phys. Rev. Lett., 96, 165002 (2006).

[20] J.T. Mendonça and J. Vieira, Phys. Plasmas, 21, 033107 (2014).

[21] J. Vieira and J.T. Mendonca, Phys. Rev. Lett., 112, 215001 (2014).

[22] R. Assmann and Awake Collaboration team, arXiv:1401.4823v2 (2014)

[23] F. Belgiorno et al., Phys. Rev. Lett., 105, 203901 (2010).

[24] E. Rubino et al., New J. Phys., 13, 085005 (2011). 